\title{
High Trait Impulsivity Predicts Food Addiction-Like Behavior in the Rat
}

\author{
Clara Velázquez-Sánchez', Antonio Ferragud', Catherine F Moore', Barry J Everitt², Valentina Sabino' and \\ Pietro Cottone*, I \\ 'Laboratory of Addictive Disorders, Departments of Pharmacology and Experimental Therapeutics and Psychiatry, Boston University School of \\ Medicine, Boston, MA, USA; ${ }^{2}$ Behavioral and Clinical Neuroscience Institute and Department of Experimental Psychology, University of \\ Cambridge, Cambridge, UK
}

\begin{abstract}
Impulsivity is a behavioral trait frequently seen not only in drug-addicted individuals but also in individuals who pathologically overeat. However, whether impulsivity predates the development of uncontrollable feeding is unknown. In this study, we hypothesized that a high impulsivity trait precedes and confers vulnerability for food addiction-like behavior. For this purpose, we trained ad libitum-fed male Wistar rats in a differential reinforcement of low rates of responding (DRL) task to select Low- and High-impulsive rats. Then, we allowed Low- and High-impulsive rats to self-administer a highly palatable diet (Palatable group) or a regular chow diet (Chow group) in I-h daily sessions, under fixed ratio (FR) I, FR3, FR5, and under a progressive ratio (PR) schedules of reinforcement. In addition, we tested the compulsiveness for food in Low- and High-impulsive rats by measuring the food eaten in the aversive, open compartment of a light/dark conflict test. Finally, we measured the expression of the transcription factor $\Delta F o s B$ in the shell and the core of the nucleus accumbens, which is a marker for neuroadaptive changes following addictive drug exposure. The data we obtained demonstrate that impulsivity is a trait that predicts the development of food addiction-like behaviors, including: (i) excessive intake, (ii) heightened motivation for food, and (iii) compulsive-like eating, when rats are given access to highly palatable food. In addition, we show that the food addiction phenotype in high impulsive subjects is characterized by an increased expression of the transcription factor $\Delta$ Fos $B$ in the nucleus accumbens shell. These results reveal that impulsivity confers an increased propensity to develop uncontrollable overeating of palatable food.

Neuropsychopharmacology (2014) 39, 2463-2472; doi:I0.I038/npp.20I4.98; published online 28 May 2014
\end{abstract}

\section{INTRODUCTION}

Pathological overeating is increasing in prevalence and is linked to reduced life expectancy and impaired quality of life (Ahima, 2009; Ahima and Lazar, 2013). The ready availability of highly palatable, energy-dense foods is recognized to be a causal factor in the development of excessive, uncontrollable overeating (Volkow et al, 2013). A growing number of behavioral and neurobiological findings from both clinical and preclinical studies provide convincing evidence that the uncontrollable intake of highly palatable foods can be regarded as an addiction-like disorder (Cottone et al, 2009; Cottone et al, 2012; Gearhardt et al, 2009; Johnson and Kenny, 2010; Smith and Robbins, 2013; Volkow et al, 2013). The core symptoms observed in binge-eating disorder, as described in the DSM-V (APA, 2013), closely match those that also define drug addiction (Smith and Robbins, 2013;

*Correspondence: Dr P Cottone, Laboratory of Addictive Disorders, Departments of Pharmacology and Experimental Therapeutics and Psychiatry, Boston University School of Medicine, 72 E Concord Street, R-618, Boston, MA 02118, USA, Tel: + 6176385662 ,

E-mail: cottone@bu.edu

Received 3 March 2014; revised 24 April 2014; accepted 24 April 2014; accepted article preview online 29 April 2014
Volkow and Wise, 2005). Indeed, the development of tolerance associated with escalating drug or food intake, larger amounts than intended of drug taken or food eaten, the loss of control over drug or food intake, and great difficulty in quitting drug use or overeating despite knowledge of adverse consequences as well as distress and dysphoria when attempting to abstain from drugs or food are hallmark diagnostic criteria of both drug addiction and binge-eating disorder (APA, 2013; Smith and Robbins, 2013; Volkow and Wise, 2005). Studies in humans have highlighted that the behavioral trait of impulsivity, defined as a predisposition towards rapid unplanned action (Chamberlain and Sahakian, 2007), is characteristic of both individuals addicted to drugs and also in individuals who pathologically overeat (Davis et al, 2011; Dawe and Loxton, 2004; Schag et al, 2013). It has been shown that impulsivity represents a vulnerability factor for compulsive cocaine and nicotine, but not heroin, seeking and taking (Belin et al, 2008). However, it has not been established whether the high impulsivity observed in forms of pathological overeating precedes or is a consequence of the onset of uncontrollable feeding behavior.

In this study, we hypothesized that a high impulsivity trait precedes and confers vulnerability for food addictionlike behavior. For this purpose, we trained ad libitum-fed 
male Wistar rats in a differential reinforcement of low rates of responding (DRL) task, and we selected Low- and Highimpulsive rats. Then we evaluated whether High-impulsive rats, exposed to the highly palatable food, were prone to develop a food addiction-like phenotype. We operationally defined in rats three food addiction-like behaviors, used as hallmarks of pathological overeating (APA, 2013; Smith and Robbins, 2013; Volkow et al, 2013): (i) The consumption of significantly larger amounts of highly palatable foods in a short period of time than most individuals in similar circumstances. We modeled this behavior by limiting access to a highly palatable diet in daily self-administration sessions. (ii) A high motivation to acquire and eat palatable food. We modeled this behavior using a progressive ratio (PR) schedule of reinforcement, in which the workload to obtain a pellet of food increased progressively within the session. (iii) The consumption of the highly palatable food in the face of negative consequences. We modeled this behavior using a light/dark conflict test, in which the food was offered in an aversive, bright compartment that normally suppresses eating.

\section{MATERIALS AND METHODS}

Further details are provided in Supplementary Information.

\section{Subjects}

Male Wistar rats $(n=47), 45$ days old on arrival (Charles River, Wilmington, MA), were triple-housed in wire-topped plastic cages in a 12-h reverse light cycle (lights off at 1100 hours) AAALAC-approved humidity-(60\%) and temperature-controlled $\left(22^{\circ} \mathrm{C}\right)$ vivarium. Procedures adhered to the National Institutes of Health Guide for the Care and Use of Laboratory Animals and the Principles of Laboratory Animal Care and were approved by the Boston University Medical Campus Institutional Animal Care and Use Committee. No experimental procedures involved food or water restriction/deprivation.

\section{DRL Task in ad libitum-Fed Rats}

The DRL procedure was adapted from previous reports (Simon et al, 2013; Sokolowski and Salamone, 1994; Wiley et al, 2000) in ad libitum-fed rats. Impulsive action, defined as the inability to withhold a response, was assessed on the last four sessions of the DRL 15-s schedule and was defined as efficiency (ratio between the rewarded responses and the total (rewarded + incorrect) responses), with higher efficiency reflecting more accurate performance, indicative of less impulsive action. The reinforcer was a 'supersaccharin' solution consisting of $1.5 \% \mathrm{w} / \mathrm{v}$ glucose and $0.4 \% \mathrm{w} / \mathrm{v}$ saccharin (Blasio et al 2012; Sabino et al 2009). Rats were ranked according to their efficiency scores, and subjects falling above the 60th percentile were assigned to the Lowimpulsive group, while subjects falling below the 40th percentile were assigned to the High-impulsive group.

\section{Spontaneous Locomotor Activity}

The analysis of locomotor reactivity to a novel environment was carried out during the first $2 \mathrm{~h}$ of the dark phase as previously shown (Belin et al, 2008). Rats were ranked according to their photocell beam breaks, and subjects falling below the 40th percentile were assigned to the Low-responder group, while subjects falling above the 60th percentile were assigned to the High-responder group ( $n=8 / 9$ group).

\section{Elevated Plus-Maze Test}

The elevated plus-maze test was performed as previously described (Cottone et al, 2007, 2008b; Dore et al, 2013a). The primary measure was the percentage of total arm time directed toward the open arms (ie, $100 \times$ open $\mathrm{arm} /$ (open arm + closed arm)), a validated index of anxiety-related behavior. Rats were ranked according to their percentage of open arm time, and subjects falling above the 60th percentile were assigned to the Low-anxiety group, while subjects falling below the 40th percentile were assigned to the High-anxiety group ( $n=8 / 9$ group).

\section{Binge-Like Eating Procedure in ad libitum-Fed Rats}

Following the DRL procedure and separation of Low- and High-impulsive rats, ad libitum-fed rats were trained in a FR1 binge-like eating procedure, as described previously (Blasio et al, 2013b; Cottone et al, 2012). Rats were assigned to either a 'Chow' control group (Low-impulsive/Chow, $n=6$; and High-impulsive/Chow, $n=8$ ) receiving $45 \mathrm{mg}$ chow pellets or a 'Palatable' group ((Low-impulsive/Palatable, $n=8$, and High-impulsive/Palatable, $n=7$ ) receiving a nutritionally complete, chocolate-flavored, high-sucrose diet (Blasio et al, 2013a). Rats were tested daily in 1-h sessions under FR1 schedule of reinforcement. After acquiring stable responding, the number of responses required to obtain one pellet was increased from FR1 to FR3 during four consecutive sessions and then from FR3 to FR5 for four additional sessions.

\section{PR Schedule of Reinforcement for Food}

Following testing under the fixed ratio (FR) schedules, rats were moved to a PR schedule of reinforcement, performed as described in Cottone et al (2008a). Sessions ended when subjects had not completed a ratio for $14 \mathrm{~min}$, with the last completed ratio defined as the breakpoint.

\section{Light/Dark Conflict Test}

The day after the last PR session rats were tested in a light/ dark conflict test. The test was performed as previously described (Cottone et al, 2012; Dore et al, 2013b). Under normal, control conditions, eating behavior is typically suppressed when a rat is in the aversive bright environment; a significant increase in food intake in spite of these adverse conditions, as compared with control conditions, was operationalized as a construct of 'compulsive-like eating'.

\section{Perfusions, Immunohistochemistry, and Quantification}

At the end of the behavioral procedures, animals were transcardially perfused as in Iemolo et al (2013). Brains were removed and processed for $\triangle$ FosB immunohistochemistry as previously described in Velazquez-Sanchez et al (2011). The brain regions of interest were the nucleus 
accumbens shell (NAcc shell) and core (NAcc core) and the dorsal striatum. The results of the quantification were expressed as the number of $\Delta$ FosB-positive cells (cell/ volume) for each of the region studied.

\section{Food Addiction-Like Score}

The food addiction-like score was calculated using a previously described approach (Deroche-Gamonet et al, 2004). Animals were ranked for each criterion independently. A subject was considered positive for a given criterion if it fell above the 67 th percentile of that distribution. The food addiction-like score was calculated as the algebraic sum of the individual standardized scores of each of the three food addiction-like behaviors (excessive intake, motivation for food, or compulsive-like eating).

\section{Statistics}

Parametrical data were analyzed by simple or factorial ANOVAs followed by Fisher's LSD tests. Statistical significance level was set at $\alpha \leqslant 0.05$. For further details, see Supplementary Information Methods.

\section{RESULTS}

\section{Impulsivity is a Phenotype Distinct from Novelty-Induced Locomotor Activity}

We first wanted to determine whether impulsivity, as measured by the tendency to respond prematurely in a DRL task, was a phenotype that is distinct from noveltyinduced locomotor activity. For this purpose, we therefore identified Low- and High-responder rats by measuring their spontaneous locomotor activity in a novel environment (Reactivity: $t_{(15)}=6.55, p \leqslant 0.0001$, Figure 1a, left panel). We then assessed the tendency to respond prematurely in Lowand High-responders by measuring the DRL efficiency, a variable which is inversely correlated with the tendency to respond prematurely (see Supplementary Materials). As shown in Figure 1a (right panel), no differences in efficiency were observed between Low- and High-responder rats (Reactivity: $t_{(15)}=0.85$, NS). Analogously, when rats were instead divided into the Low- and High-impulsive groups based on their tendency to respond prematurely in the DRL (Impulsivity: $t_{(15)}=8.94, p \leqslant 0.0001$, Figure $1 \mathrm{~b}$, left panel), no differences in spontaneous locomotor activity in a novel environment were observed between groups (Impulsivity: $t_{(15)}=1.52$, NS, Figure 1b, right panel).

\section{Impulsivity is a Phenotype Distinct from Anxiety-Like Behavior}

To determine whether impulsivity was a phenotype different from anxiety-like behavior, we first identified Low- and High-anxiety rats by measuring their spontaneous anxiety-like behavior in an elevated plus-maze test (Anxiety: $t_{(16)}=10.98, p \leqslant 0.0001$, Figure $1 c$, left panel). We then measured the DRL efficiency in Low- and High-anxiety rats and, as shown in Figure 1c right panel, and no differences in efficiency were observed between groups (Impulsivity: $t_{(16)}=0.42$, NS). Similarly, when rats were instead divided into the Low- and High-impulsive groups based on their tendency to respond prematurely in the DRL (Figure 1b, left panel), no differences in anxiety-like behavior were
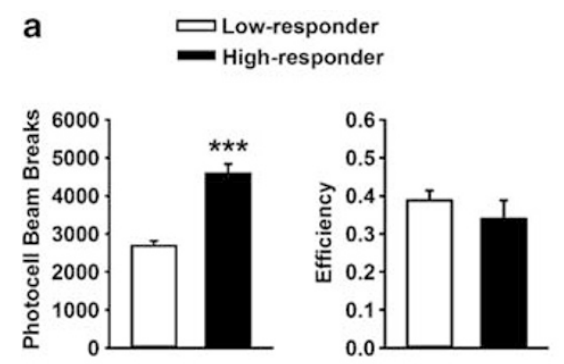

b

$\underset{\text { Low-impulsive }}{\square \text { High-impulsive }}$
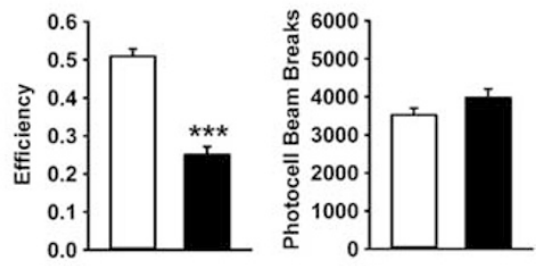

C $\quad$ Low-anxiety

d
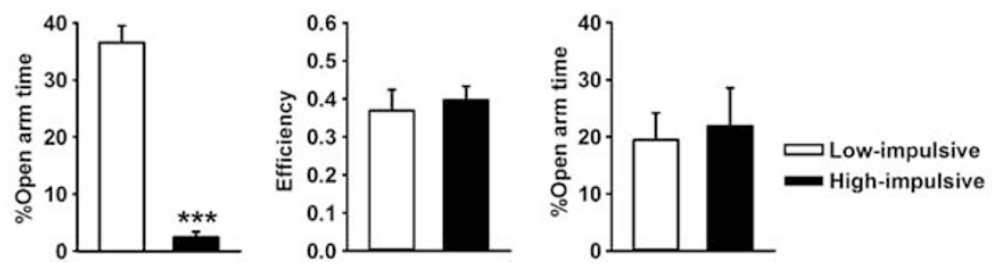

Figure I Impulsivity is a phenotype distinct from both novelty-induced locomotor activity and anxiety-like behavior. (a) Left: Screening for Low- and Highresponsive rats based on spontaneous locomotor reactivity to novelty. High-responsive rats show higher locomotor activity than Low-responsive rats. (a) Right: Low- and High-responsive rats do not differ in their tendency to respond prematurely in the DRL task. (b) Left: Screening for Low- and Highimpulsive rats using a DRL task. High-impulsive rats are less efficient in responding compared with Low-impulsive rats. (b) Right: Low- and High-impulsive rats do not differ in novelty-induced locomotor activity. (c) Left: Screening for Low- and High-anxiety rats based on the percentage of open arm time in the elevated plus-maze. High-anxiety rats show lower percentage of open arm time than Low-anxiety rats. (c) Right: Low- and High-anxiety rats do not differ in their tendency to respond prematurely in the DRL task. (d) Low- and High-impulsive rats (screened in panel (b) left) do not differ in anxiety-like behavior. $n=8 / 9$ group. Data show $M \pm S E M$. ***** $p \leqslant 0.000$ I vs Low counterpart. 
observed between groups (Impulsivity: $t_{(16)}=0.30$, NS, Figure 1d).

\section{High Trait Impulsivity Predicts the Escalation of Responding for Highly Palatable Food}

To test the hypothesis that high impulsivity predisposes to food addiction-like behavior, we identified Low- and Highimpulsive rats by measuring their efficiency (Impulsivity: $\mathrm{F}_{(1,25)}=31.705, p \leqslant 0.0001$, Figure $\left.2 \mathrm{a}\right)$. Then both Low- and High-impulsive rats were trained in a validated binge-like eating procedure (Blasio et al, 2013b; Cottone et al, 2012) based on a limited access to a highly palatable food. Thus, half of the rats were provided with daily 1-h access to a highly palatable, sugary diet (Low-impulsive/Palatable and High-impulsive/Palatable), while the other half was given a regular chow diet (Low-impulsive/Chow and Highimpulsive/Chow) (Blasio et al, 2013b; Cottone et al, 2012). Our results show that during their very first experience with the novel highly palatable diet, Low-impulsive/Palatable rats, which were otherwise eating the regular chow diet, rejected as expected the newly provided food, as result of a neophobic response (Figure 2b). Conversely, Highimpulsive/Palatable rats showed no neophobia and readily accepted the new diet, showing no change in the amount of food consumed compared with Chow control rats (Impulsivity: $F_{(1,25)}=6.523, p \leqslant 0.02$ ). With further daily access to the highly palatable diet, all rats progressively increased their intake compared with control chow-fed rats; however, as predicted, the High-impulsive/Palatable rats overate the highly palatable food to a much greater extent than Low-impulsive/Palatable rats, ingesting up to four times the intake of chow-fed control rats (Impulsivity $\times$ Food: $\mathrm{F}_{(1,25)}=6.25, p \leqslant 0.02$ ) (Figure $2 \mathrm{c}$ ). No effect in water responding was observed (Impulsivity $\times$ Food: $F_{(1,25)}=0.03$, NS, not shown).

\section{High Trait Impulsivity Predicts an Increased Motivation for Highly Palatable Food}

Subsequently, we sought to determine whether high trait impulsivity is a factor that heightens the motivation for palatable food. To test this hypothesis, in the same cohort of rats, following the escalation of palatable food intake and establishment of a stable baseline of responding for food under a FR1 schedule of reinforcement, we increased the price (number of responses) required to obtain each food pellet. The results show that increasing the workload required to obtain the food (from FR1 to FR5) resulted in decreased responding by Low-impulsive/Palatable rats, whereas High-impulsive/Palatable rats maintained the highest level of responding compared with all the other groups (FR3 Figure 2d left, Impulsivity: $\mathrm{F}_{(1,25)}=6.37, p \leqslant 0.02$; Food: $\mathrm{F}_{(1,25)}=23.91, p \leqslant 0.00005$; FR5 Figure $2 \mathrm{~d}$ right, Food: $\quad \mathrm{F}_{(1,25)}=24.778, \quad p \leqslant 0.00005 ; \quad$ Impulsivity $\times$ Food: $\left.\mathrm{F}_{(1,25)}=4.33, p \leqslant 0.05\right)$. As responding for food among groups varied as a function of the response requirement, we next determined the maximum effort each rat was willing to make to obtain a single food unit pellet, using a PR schedule task (Belin et al, 2008; Deroche-Gamonet et al, 2004). In the PR task, the number of responses required to obtain each food pellet progressively increased within each session until the rat reached a point beyond which it was no longer willing to respond for food. The maximum amount of work that the rat performed before cessation of responding, referred to as the breakpoint, is not influenced by satiety factors and is considered a reliable index of motivation (Belin et al, 2008; Deroche-Gamonet et al, 2004; Hodos, 1961). As predicted, High-impulsive/Palatable rats showed significantly higher breakpoints than all the other groups, while Low-impulsive/Palatable rats did not differ in their motivation to obtain food compared with chow-fed control rats (Food: $\mathrm{F}_{(1,25)}=13.91, p \leqslant 0.001$; Figure $2 \mathrm{e}$ ).

\section{High Trait Impulsivity Predicts Compulsive-Like Eating of Highly Palatable Food}

Next, we determined whether high impulsivity predicted the loss of control over palatable food intake and compulsivelike eating. To test this hypothesis, we tested the rats in a light/dark conflict test, in which the food was offered in the aversive, bright compartment of the apparatus (Cottone et al, 2012; Dore et al, 2013b). Therefore, rats were required to face the aversive context in order to consume the food. Regular chow consumption by Low- and High-impulsive/ Chow rats was negligible in the aversive context. The Low-impulsive/Palatable rats ate an amount of food not significantly different compared with the food consumed by both the Chow groups (Figure 2f). By contrast, Highimpulsive/Palatable rats ate a significantly greater amount of palatable food compared with any other group ( $\sim 22-$, $\sim 38$-, and $\sim 3$-fold increase as compared with Lowimpulsive/Chow, High-impulsive/Chow and Low-impulsive/ Palatable rats, respectively; Food: $\left.\mathrm{F}_{(1,25)}=8.19, p \leqslant 0.01\right)$.

\section{High Trait Impulsivity Predicts a Food Addiction-Like Phenotype}

To further clarify the influence of impulsivity on the individual susceptibility to develop a food addictive-like phenotype, we took a similar approach to that used previously (Belin et al, 2008; Deroche-Gamonet et al, 2004) to calculate an 'addiction score', in which a rat was considered positive for each of the three established food addiction-like criteria ((i) excessive intake, (ii) heightened motivation for food, and (iii) compulsive-like eating), if it fell above the 33th percentile of the distribution. This approach allowed the division of our sample of rats into four groups according to the number of positive criteria met (zero, one, two, or three) (Belin et al, 2008; DerocheGamonet et al, 2004). As shown in Figure 3, the intensity of the three food addiction-like behaviors was proportional to the number of positive criteria met by each subject (Figure $3 a-c)$. Furthermore, we defined the food addictionlike score as the sum of the standardized scores of the three criteria to represent the overall contribution of the three food addiction-like behaviors in a single score (Belin et al, 2008). The food addiction-like score highly correlated with excessive intake $\left(r^{2}=0.74, p \leqslant 0.000001\right)$, motivation for food $\left(r^{2}=0.60, p \leqslant 0.00001\right)$, and compulsive-like eating $\left(r^{2}=0.60, p \leqslant 0.00001\right)$ (Figure $\left.3 \mathrm{~d}-\mathrm{f}\right)$. Rats belonging to zero, one, two, or three positive criteria groups were linearly distributed along the food addiction-like score, with the three criteria group showing the highest food addiction-like 

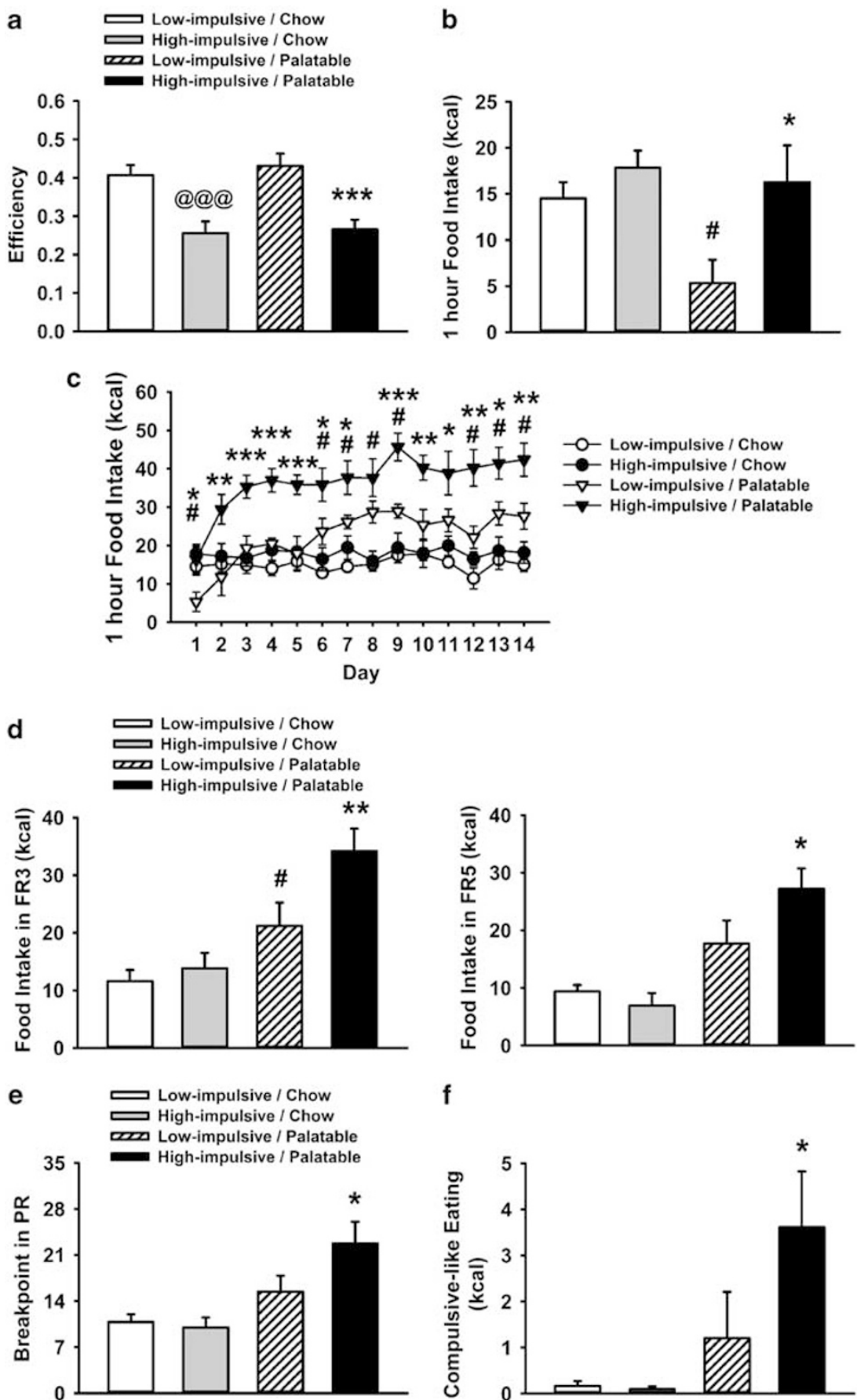

Figure 2 High trait impulsivity predicts the disruption of neophobia, the escalation of highly palatable food intake, the motivation for, and the compulsivelike eating of highly palatable food. (a) Screening for Low- and High-impulsive rats using a DRL task. High-impulsive rats are less efficient in responding compared with Low-impulsive rats. (b) One-hour food intake during the first day of palatable food self-administration in Low- and High-impulsive rats. Expectedly Low-impulsive/Palatable rats showed food neophobia, contrary to High-impulsive/Palatable rats who readily accepted the new diet. (c) Effects of daily, I-h access to a highly palatable diet on food intake in Low- and High-impulsive rats. High-impulsive/Palatable rats dramatically escalated responding for the highly palatable diet, resulting in a significantly higher food intake compared with all other groups. (d) Effects of daily, one-hour access to a highly palatable diet on food intake under FR3 and FR5 schedules of reinforcement. High-impulsive/Palatable rats showed higher responding for food compared with all other groups. (e) Motivation for food, measured as the breakpoint under a progressive-ratio schedule of reinforcement. High-impulsive/Palatable rats showed higher breakpoint for food than all the other groups. (f) Compulsive-like eating behavior measured in the light/dark conflict test. High-impulsive/Palatable rats showed higher food intake compared with all other groups. $n=29$. Data show mean \pm SEM. @@@ $p \leqslant 0.00$ I High-impulsive/Chow vs Low-impulsive/Chow; $p \leqslant 0.05$, Low-impulsive/Palatable vs Low-impulsive/Chow; $* \leqslant 0.0$ I, *** $p \leqslant 0.00$ I, **** $p \leqslant 0.000$ I High-impulsive/Palatable vs Low-impulsive/Palatable.

score (Figure 4a). More importantly, as shown in Figure 4b, high trait impulsivity predicted a heightened food addiction-like behavior in rats exposed to the highly palatable diet, as the food addiction score in High-impulsive/ Palatable rats was higher than that of all the other groups (Impulsivity $\times$ Food: $\left.F_{(1,25)}=9.54, p \leqslant 0.005\right)$. Figure $4 \mathrm{c}$ 

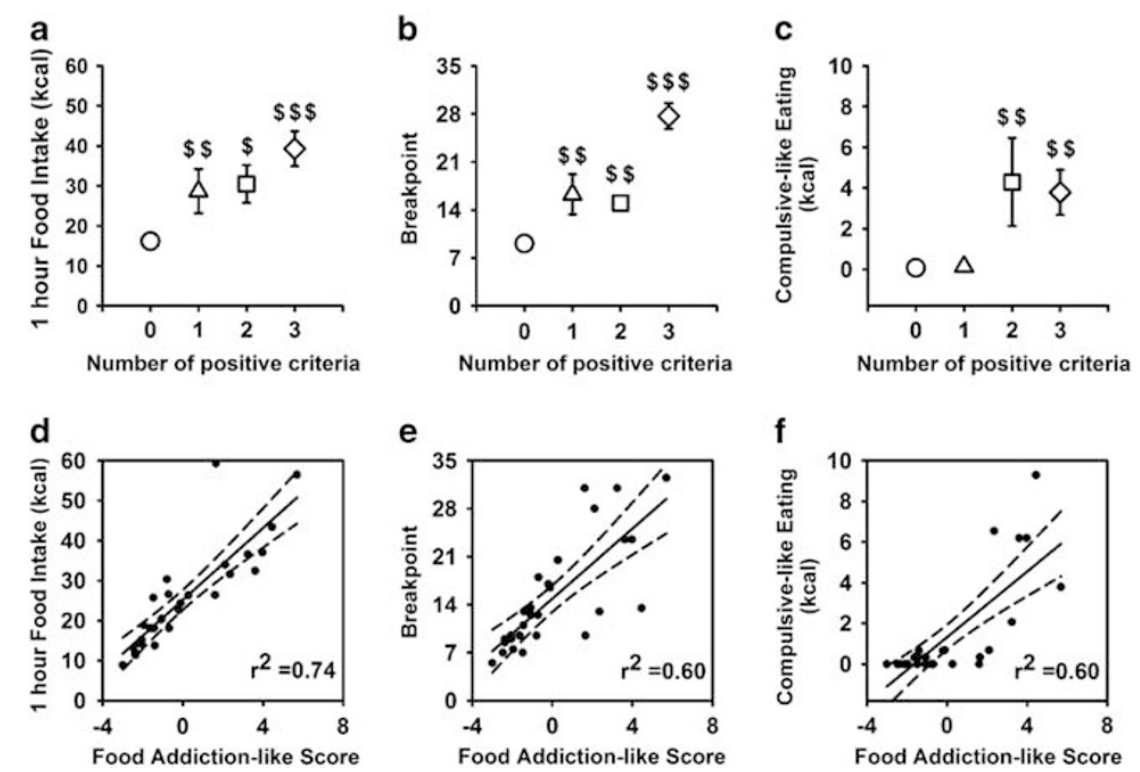

Figure 3 Food addiction-like score highly correlates with escalated intake, motivation for food, and compulsive-like eating. (a-c) Excessive intake, motivation for food, and compulsive-like eating as a function of the number of positive food addiction-like criteria met. The intensity of the three food addiction-like behaviors was proportional to the number of positive criteria met by the subjects. ( $d-f$ ) The food addiction-like score highly correlated with excessive intake, motivation for food, and compulsive-like eating. $n=29$. Data show mean \pm SEM. ${ }^{\$} p \leqslant 0.05,{ }^{\$ \$} p \leqslant 0.0$ I, ${ }^{\$ \$ \$} p \leqslant 0.00$ I vs zero criteria group.
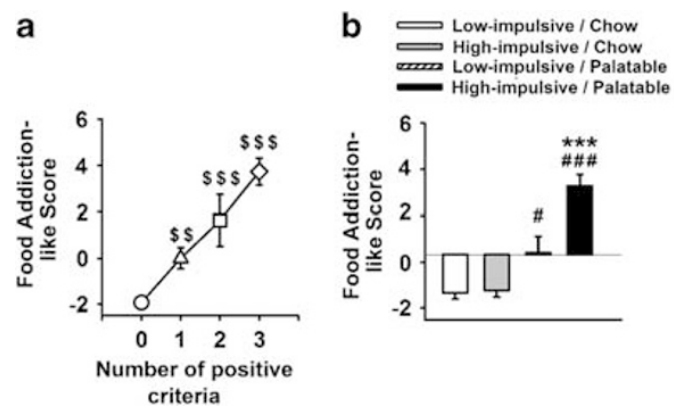

Figure 4 Three criteria and High-impulsive/Palatable rats show highest food addiction-like score. (a) Rats with the three criteria showed a food addictionlike score $(3.72 \pm 0.47)$ above the SD of all rats $(2.4 I)$ and higher than any other groups. (b) Food addiction-like score as function of the four experimental groups. The food addiction-like score in High-impulsive/Palatable rats was higher than in all other. (c) Percentage of the total population of Low- and Highimpulsive/Chow and Palatable rats positive for zero, one, two, or three food addiction-like criteria. $n=29$. Data show mean \pm SEM. ${ }^{\$ \$} p \leqslant 0.01,{ }^{\$ \$ \$} p \leqslant 0.00$ I vs zero criteria group; ${ }^{\#} \leqslant 0.05 ;{ }^{\# \# \# ~} p \leqslant 0.00$ I vs Low-impulsive/Chow; ${ }^{*} * * * \leqslant 0.00$ I vs Low-impulsive/Palatable.

shows the percentage of the total population of Low- and High-impulsive/Chow and Palatable rats positive for zero, one, two, or three food addiction-like criteria. Notably, the proportion of Palatable rats increased as a function of the number of positive criteria met either when data were analyzed independently from the impulsivity factor (15.4, 57.1, 75.0, and $100.0 \%$ in zero, one, two, and three criteria group, respectively) or when only high-impulsive subjects were taken into account $(0,50.0,100.0$, and $100.0 \%$ in zero, one, two, and three criteria group, respectively). Therefore, three criteria group was exclusively composed of rats trained with the palatable diet and $80.0 \%$ of these were high impulsive. On the other hand, zero criteria group was mostly composed of Chow rats $(84.6 \%)$, which were equally represented in the low and high impulsivity trait.

\section{$\Delta$ FosB Expression in the Shell of the NAcc is a Molecular Marker of Food Addiction-Like Behavior}

Finally, we investigated whether $\Delta \mathrm{FosB}$, a well-characterized transcription factor, the induction of which in the NAcc is a marker for neuroadaptive changes to addictive drug use (Nestler, 2008), was correlated with food addiction-like behaviors in high impulsive rats (Nestler, 2012). We undertook quantitative analysis of $\Delta$ FosB immunoreactivity in the NAcc shell and core (Figure 5) and dorsal striatum (Supplementary Figure S1) of the same rats used for the behavioral studies. As shown in Figure 5, exposure to the highly palatable diet was associated with an overall significant increase of $\Delta$ FosB-positive cell counts in the two subregions of the NAcc, the shell and the core (core, 

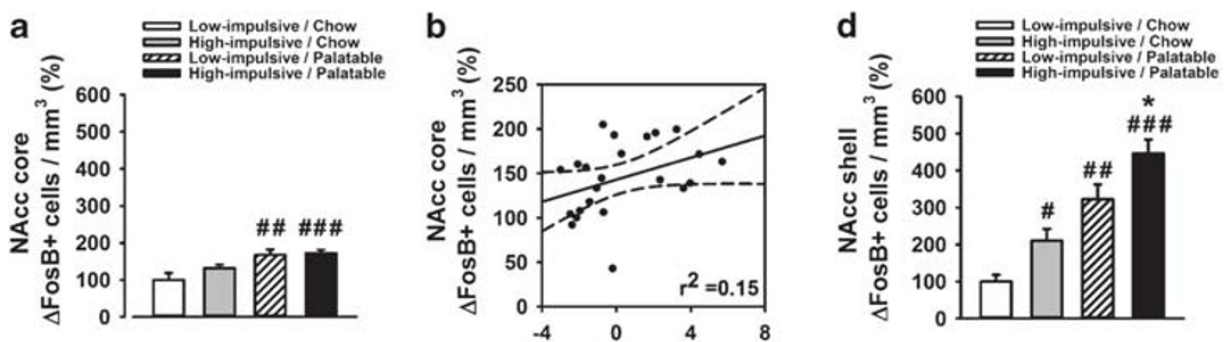

Food Addiction-like Score

c

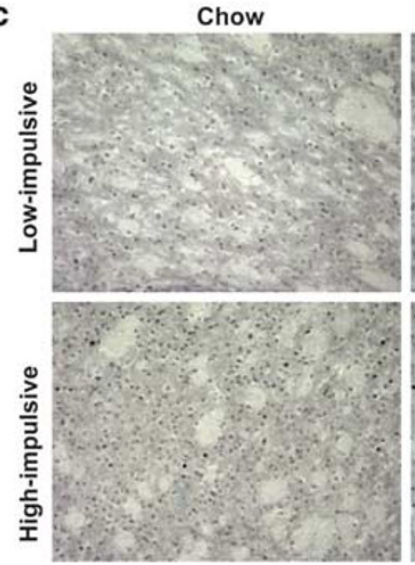

Palatable

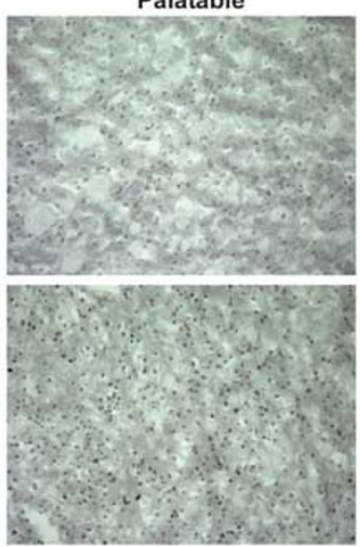

$\mathbf{f}$

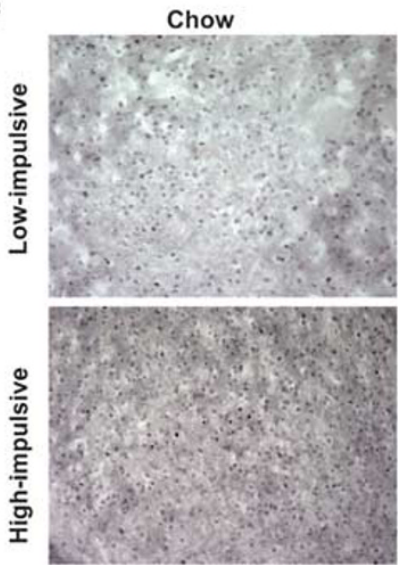

e

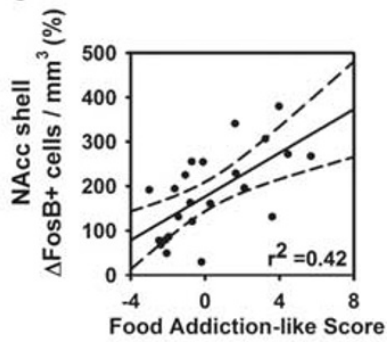

Palatable

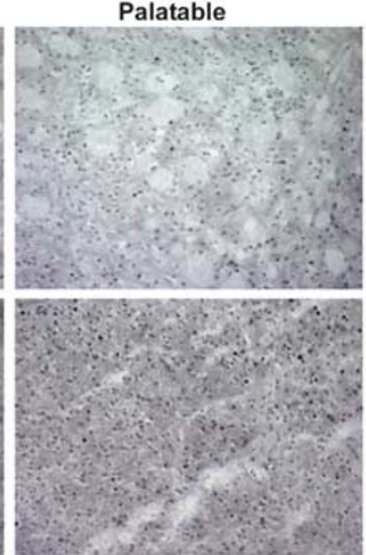

Figure $5 \quad \Delta$ FosB expression in the nucleus accumbens (NAcc). (a and d) $\Delta$ FosB expression in the NAcc core and shell expressed as density change (\%) in relation to the Low-impulsive/Chow group. High-impulsive/Palatable animals showed a significant increase in both regions compared with the Lowimpulsive/Chow group and a significant increase in the NAcc core compared with the Low-impulsive/Palatable. (b and e) Food addiction-like behavior highly correlated with $\Delta$ FosB expression in the NAcc shell but not in the NAcc core. (c and $f$ ) Representative micrographs $(\times 20)$ of $\Delta$ FosB expression in NAcc core and shell of the different groups are shown. $n=23$. Data show mean \pm SEM. ${ }^{*} p \leqslant 0.05,{ }^{\# \#} p \leqslant 0.01,{ }^{\# \# \#} p \leqslant 0.00$ I vs Low-impulsive/Chow; ${ }^{*} p \leqslant 0.05$ vs Low-impulsive/Palatable.

Food: $\mathrm{F}_{(1,19)}=17.60, p \leqslant 0.0005$; shell, Food: $\mathrm{F}_{(1,19)}=39.54$, $p \leqslant 0.000005$; Figure 5a, c, d, and f). However, in the NAcc shell a strong effect of impulsivity was observed (shell, Impulsivity: $\left.\mathrm{F}_{(1,19)}=10.27, p \leqslant 0.005\right)$, so that both the High-impulsive/Chow and High-impulsive/Palatable groups showed higher $\Delta$ FosB-positive cell counts as compared with the respective Low-impulsive counterparts. As a result, the additive effects of Food and Impulsivity induced a fourfold increase of $\triangle \mathrm{FosB}$ immunoreactive staining in Highimpulsive/Palatable rats compared with Low-impulsive/ Chow and which was higher than all other groups (Figure 5d). A highly significant correlation between the $\Delta$ FosB expression and the food addiction-like score was found in the NAcc shell $\left(r^{2}=0.42, p \leqslant 0.0009\right.$, Figure 5e) but not in the core $\left(r^{2}=0.15\right.$, NS, Figure $\left.5 b\right)$. The analysis of the dorsal striatum revealed an effect of the exposure to the diet (Food: $\mathrm{F}_{1,20}=9.5, p \leqslant 0.05$ ) given by a weak $\Delta$ FosB immunoreactive staining increase in High-impulsive/Palatable rats compared with Low-impulsive/Chow. The $\Delta$ FosB expression in the dorsal striatum was weakly correlated with the food addiction-like score $\left(r^{2}=0.21, p \leqslant 0.05\right)$ (Supplementary Figure S1).

\section{DISCUSSION}

These results provide experimental evidence that the higher tendency to respond prematurely, one dimension of the complex construct of impulsivity (Dalley et al, 2008;
Evenden, 1999), is a behavioral trait that predisposes individuals to food addiction-like behavior. Our preclinical data extend clinical findings showing that individuals who binge eat show heightened impulsivity and that loss of control over food intake experienced by binge-eating individuals during binge episodes is influenced by impulsivity (Davis et al, 2011; Dawe and Loxton, 2004; Schag et al, 2013).

We also provide evidence that highly palatable food interacts with preexisting high impulsivity to result in an addictive-like phenotype in rats. Although the existence of food addiction is still a debated topic (Avena et al, 2012; Corwin and Grigson, 2009; Ziauddeen et al, 2012), our results show evidence that a food addiction-like phenotype can indeed develop following exposure to palatable food, using a approach similar to the one used to prove cocaine addiction-like behavior in the rat (Deroche-Gamonet et al, 2004). This phenotype is characterized by excessive food consumption within brief periods of time, heightened motivation for food, and loss of control over eating in an adverse environment. Importantly, we demonstrate that in the sample of rats studied here there are individual differences in the addiction-like behavioral response that follows the exposure to highly palatable food. This aspect has high translational relevance, because it demonstrates that not all subjects exposed to the addictive palatable food respond likewise, analogously to what observed in Western societies where highly palatable foods are omnipresent but only some individuals develop pathological overeating 
(Davis et al, 2011; Gearhardt et al, 2009; Smith and Robbins, 2013; Volkow and Wise, 2005). Remarkably, our results reveal that high trait impulsivity is a risk factor, which can predict the individual susceptibility to the addictive properties of highly palatable foods.

In this study, we replicated the observation that high impulsive rats do not display high locomotor reactivity to novelty (Belin et al, 2008), confirming also clinical observations that measures of sensation seeking are not readily associated with disordered eating ((Fischer, 2007; Lopez-Pantoja et al, 2012; Mizushima et al, 1998); see Supplementary References 28 to 41).

Rats selected for high trait impulsivity, when exposed for the first time to the highly palatable diet, ate no differently than control chow rats. By contrast, Low-impulsive/ Palatable ate significantly less than both Low-impulsive/ Chow and High-impulsive/Palatable rats, suggesting a greater neophobic response. Neophobia is an adaptive response that protects individuals from the possible harmful postingestive consequences of unfamiliar tastants (Birch, 1999; Corey, 1978). Therefore, the high impulsivity trait is associated with the disruption of this physiological, defensive mechanism in response to unknown tastants. It has been proposed that neophobia is a measure of anxiety (Britton and Britton, 1981; Hall, 1934), raising the potential alternative interpretation that the observed differences in the food-related variables throughout this study may be influenced by an increased anxiety-like trait in low impulsive rats. However, we can confidently exclude this alternative interpretation for several reasons. First, replicating previous observations with the 5-CSRTT (Molander et al, 2011), we have found that low and high impulsive rats, selected based on their tendency to respond prematurely in a DRL task, do not differ in their anxiety-like behavior, and therefore impulsivity and anxiety-like behavior are distinct phenotypes. Furthermore, our previous work demonstrated that neophobia does not reflect a single behavioral process and does not represent a pure index of anxiety or reactivity to novelty (Cole et al, 1988). Finally, the neophobic response was observed only during the first exposure to the highly palatable diet; by the second exposure, Low-impulsive/Palatable rats ate similarly to Low-impulsive/Chow rats, and by the sixth day of access Low-impulsive/Palatable rats were eating significantly more than chow control rats. Taken together, these data indicate that impulsivity in rats, measured using the DRL, which predicts vulnerability for food addiction-like behavior, is distinct from anxiety.

We analyzed the levels of the transcription factor $\Delta \mathrm{FosB}$ in the NAcc shell and core of low and high impulsive rats exposed to the regular chow or the highly palatable food, as $\Delta$ FosB has a key role in the neuroplastic changes following repeated exposure to addictive drugs (Nestler, 2008). Remarkably, we observed that high impulsive rats, which were exposed to the regular chow diet, showed increased $\Delta$ FosB levels in the shell of the NAcc compared with the low impulsive counterpart. This significant result suggests that $\Delta$ FosB in the NAcc shell is a marker of the high impulsivity trait, independent of access to the highly palatable diet. In addition, confirming the role of $\Delta$ FosB in the response to exposure to natural rewards (Olausson et al, 2006; Sharma et al, 2013; Teegarden et al, 2009; Wallace et al, 2008;
Werme et al, 2002), we observed a strong effect of exposure to the highly palatable diet on $\Delta$ FosB levels in the same brain region, independent of the factor impulsivity. Therefore, the contribution of each of the two factors resulted in a robust increase of $400 \%$ in $\Delta \mathrm{FosB}$ level in High-impulsive/ Palatable rats as compared with those of Low-impulsive/ Chow rats. Importantly, the $\Delta$ FosB expression in the NAcc shell highly correlated with the food addiction-like score.

We can exclude that any potential energy homeostasis confounding effects played any role on the observed food addiction-like phenotype. First, the reinforcer in the DRL task is a solution, the caloric content of which is negligible $(0.006 \mathrm{Kcal})$, and the reinforcing efficacy of which is mostly driven by its non-caloric component, saccharin (see Supplementary Material). Therefore, against a potential selection of rats based on energy needs, rather than impulsivity, the total energy intake upon completion of a DRL session was merely $0.28 \pm 0.01 \mathrm{Kcal}(\mathrm{M} \pm \mathrm{SEM})$, a value representing $<0.3 \%$ of a rat's typical daily caloric intake. In addition, against a potential selection of rats based on energy needs, Low- and High-impulsive/Chow rats never differed in responding for food during the FR1 escalation. Finally, Low- and High-impulsive/Chow rats never differed in body weight during the study, therefore excluding any potential energy balance confounding factor on the observed feeding adaptations (Blasio et al, 2013b; Cottone et al, 2012). Finally, all the behavioral tasks in this study were designed in such a way that rats were never food restricted or deprived, therefore excluding any potential influence of an altered energy balance state. This aspect is particularly relevant in the context of the DRL task; to the best of our knowledge, there is no model of impulsive action that has been designed to be performed in ad libitum-fed rats. Indeed, the use of food restriction/deprivation to motivate rats to perform in impulsivity tasks represents a severe limitation in experiments in which food consumption in a non-deprived state is an experimental variable. Therefore, our DRL task overcomes one of the major challenges in the study of impulsivity in the context of feeding behavior.

The approach taken in this study is analogous to the one used previously showing that high impulsivity predicts compulsive cocaine taking (Belin et al, 2008); however, the two approaches also embody important differences to the operationalization of the addiction-like phenotype, primarily due to the different pathology investigated. Indeed, unlike that used by Belin et al (2008), we integrated the construct of 'excessive intake within a brief period of time' within the food addiction-like phenotype, as this behavior represents the fundamental core symptom of the pathology we modelled in this study (APA, 2013).

From an evolutionary perspective, we speculate that, in environments where starvation threatened survival, impulsivity may have played an advantageous, adaptive role by enhancing reward sensitivity and shifting decision-making towards less planned responses for the selection of more palatable and energy-dense foods. However, the same neurobiological mechanism, in today's calorie-rich environment, may instead promote pathological overeating. The data we report in this study have therefore translational significance and provide insights into the neurobiological mechanisms underlying uncontrollable food intake. 


\section{FUNDING AND DISCLOSURE}

This publication was made possible by grant numbers DA030425, MH091945, and MH093650 from the National Institute on Drug Abuse (NIDA), the National Institute of Mental Health (NIMH), by the Peter Paul Career Development Professorship (to PC), the McManus Charitable Trust (to VS), and Boston University's Undergraduate Research Opportunities Program (UROP). Its contents are solely the responsibility of the authors and do not necessarily represent the official views of the National Institutes of Health. The authors declare no conflict of interest.

\section{ACKNOWLEDGEMENTS}

We thank Stephen St Cyr for technical assistance and Alyssa DiLeo and Anika Begum for editorial assistance.

\section{REFERENCES}

APA (2013). Diagnostic and Statistical Manual of Mental Disorders. 5th edn. American Psychiatric Association.

Ahima RS (2009). The end of overeating: taking control of the insatiable American appetite. J Clin Invest 119: 2867.

Ahima RS, Lazar MA (2013). Physiology. The health risk of obesity-better metrics imperative. Science 341: 856-858.

APA (2013). Diagnostic and Statistical Manual of Mental Disorders,. 5th edn. American Psychiatric Association.

Avena NM, Gearhardt AN, Gold MS, Wang GJ, Potenza MN (2012). Tossing the baby out with the bathwater after a brief rinse? The potential downside of dismissing food addiction based on limited data. Nat Rev Neurosci 13: 514 author reply 514.

Belin D, Mar AC, Dalley JW, Robbins TW, Everitt BJ (2008). High impulsivity predicts the switch to compulsive cocaine-taking. Science 320: 1352-1355.

Birch LL (1999). Development of food preferences. Annu Rev Nutr 19: 41-62.

Blasio A, Iemolo A, Sabino V, Petrosino S, Steardo L, Rice KC et al (2013a). Rimonabant precipitates anxiety in rats withdrawn from palatable food: role of the central amygdala. Neuropsychopharmacology 38: 2498-2507.

Blasio A, Narayan AR, Kaminski BJ, Steardo L, Sabino V, Cottone P (2012). A modified adjusting delay task to assess impulsive choice between isocaloric reinforcers in non-deprived male rats: effects of $5-\mathrm{HT}_{2} \mathrm{~A} / \mathrm{C}$ and $5-\mathrm{HT}_{1} \mathrm{~A}$ receptor agonists. Psychopharmacology (Berl) 219: 377-386.

Blasio A, Steardo L, Sabino V, Cottone P (2013b). Opioid system in the medial prefrontal cortex mediates binge-like eating. Addict Biol (doi:10.1111/adb.12033).

Britton DR, Britton KT (1981). A sensitive open field measure of anxiolytic drug activity. Pharmacol Biochem Behav 15: 577-582.

Chamberlain SR, Sahakian BJ (2007). The neuropsychiatry of impulsivity. Curr Opin Psychiatry 20: 255-261.

Cole BJ, Robbins TW, Everitt BJ (1988). Lesions of the dorsal noradrenergic bundle simultaneously enhance and reduce responsivity to novelty in a food preference test. Brain Res 472: 325-349.

Corey DT (1978). The determinants of exploration and neophobia. Neurosci Biobehav Rev 2: 235-253.

Corwin RL, Grigson PS (2009). Symposium overview-food addiction: fact or fiction? J Nutr 139: 617-619.

Cottone P, Sabino V, Roberto M, Bajo M, Pockros L, Frihauf JB et al (2009). CRF system recruitment mediates dark side of compulsive eating. Proc Natl Acad Sci USA 106: 20016-20020.

Cottone P, Sabino V, Steardo L, Zorrilla EP (2007). FG 7142 specifically reduces meal size and the rate and regularity of sustained feeding in female rats: evidence that benzodiazepine inverse agonists reduce food palatability. Neuropsychopharmacology 32: 1069-1081.

Cottone P, Sabino V, Steardo L, Zorrilla EP (2008a). Intermittent access to preferred food reduces the reinforcing efficacy of chow in rats. Am J Physiol 295: R1066-R1076.

Cottone P, Sabino V, Steardo L, Zorrilla EP (2008b). Opioiddependent anticipatory negative contrast and binge-like eating in rats with limited access to highly preferred food. Neuropsychopharmacology 33: 524-535.

Cottone P, Wang X, Park JW, Valenza M, Blasio A, Kwak J et al (2012). Antagonism of sigma-1 receptors blocks compulsive-like eating. Neuropsychopharmacology 37: 2593-2604.

Dalley JW, Mar AC, Economidou D, Robbins TW (2008). Neurobehavioral mechanisms of impulsivity: fronto-striatal systems and functional neurochemistry. Pharmacol Biochem Behav 90: 250-260.

Davis C, Curtis C, Levitan RD, Carter JC, Kaplan AS, Kennedy JL (2011). Evidence that 'food addiction' is a valid phenotype of obesity. Appetite 57: 711-717.

Dawe S, Loxton NJ (2004). The role of impulsivity in the development of substance use and eating disorders. Neurosci Biobehav Rev 28: 343-351.

Deroche-Gamonet V, Belin D, Piazza PV (2004). Evidence for addiction-like behavior in the rat. Science 305: 1014-1017.

Dore R, Iemolo A, Smith KL, Wang X, Cottone P, Sabino V (2013a). CRF mediates the anxiogenic and anti-rewarding, but not the anorectic effects of PACAP. Neuropsychopharmacology 38: 2160-2169.

Dore R, Valenza M, Wang X, Rice KC, Sabino V, Cottone P (2013b). The inverse agonist of CB receptor SR141716 blocks compulsive eating of palatable food. Addict Biol (doi:10.1111/adb.12056).

Evenden JL (1999). Varieties of impulsivity. Psychopharmacology (Berl) 146: 348-361.

Fischer S (2007). The relationship of neuroticism and urgency to negative consequences of alcohol use in women with bulimic symptoms. Pers Individual Differences 43: 1199-1209.

Gearhardt AN, Corbin WR, Brownell KD (2009). Preliminary validation of the Yale Food Addiction Scale. Appetite 52: 430-436.

Hall CS (1934). Emotional behavior in the rat. I. Defecation and urination as measures of individual differences in emotionality. J Comp Psychol 18: 385-403.

Hodos W (1961). Progressive ratio as a measure of reward strength. Science 134: 943-944.

Iemolo A, Blasio A, St Cyr SA, Jiang F, Rice KC, Sabino V et al (2013). CRF-CRF1 receptor system in the central and basolateral nuclei of the amygdala differentially mediates excessive eating of palatable food. Neuropsychopharmacology 38: 2456-2466.

Johnson PM, Kenny PJ (2010). Dopamine D2 receptors in addiction-like reward dysfunction and compulsive eating in obese rats. Nat Neurosci 13: 635-641.

Lopez-Pantoja JL, Cabranes JA, Sanchez-Quintero S, Velao M, Sanz M, Torres-Pardo B et al (2012). Personality profiles between obese and control subjects assessed with five standardized personality scales. Actas Esp Psiquiatr 40: 266-274.

Mizushima H, Ono Y, Asai M (1998). TCI temperamental scores in bulimia nervosa patients and normal women with and without diet experiences. Acta Psychiatr Scand 98: 228-230.

Molander AC, Mar A, Norbury A, Steventon S, Moreno M, Caprioli D et al (2011). High impulsivity predicting vulnerability to cocaine addiction in rats: some relationship with novelty preference but not novelty reactivity, anxiety or stress. Psychopharmacology (Berl) 215: 721-731.

Nestler EJ (2008). Review. Transcriptional mechanisms of addiction: role of DeltaFosB. Philos Trans R Soc Lond Ser B Biol Sci 363: 3245-3255.

Nestler EJ (2012). Transcriptional mechanisms of drug addiction. Clin Psychopharmacol Neurosci 10: 136-143. 
High impulsivity predicts food addiction

C Velázquez-Sánchez et al

Olausson P, Jentsch JD, Tronson N, Neve RL, Nestler EJ, Taylor JR (2006). DeltaFosB in the nucleus accumbens regulates food-reinforced instrumental behavior and motivation. J Neurosci 26: 9196-9204.

Sabino V, Cottone P, Zhao Y, Iyer MR, Steardo L Jr., Steardo L et al (2009). The sigma-receptor antagonist BD-1063 decreases ethanol intake and reinforcement in animal models of excessive drinking. Neuropsychopharmacology 34: 1482-1493.

Schag K, Schonleber J, Teufel M, Zipfel S, Giel KE (2013). Foodrelated impulsivity in obesity and binge eating disorder-a systematic review. Obes Rev 14: 477-495.

Sharma S, Fernandes MF, Fulton S (2013). Adaptations in brain reward circuitry underlie palatable food cravings and anxiety induced by high-fat diet withdrawal. I J Obes (Lond) 37: 1183-1191.

Simon NW, Beas BS, Montgomery KS, Haberman RP, Bizon JL, Setlow B (2013). Prefrontal cortical-striatal dopamine receptor mRNA expression predicts distinct forms of impulsivity. Eur $J$ Neurosci 37: 1779-1788.

Smith DG, Robbins TW (2013). The neurobiological underpinnings of obesity and binge eating: a rationale for adopting the food addiction model. Biol Psychiatry 73: 804-810.

Sokolowski JD, Salamone JD (1994). Effects of dopamine depletions in the medial prefrontal cortex on DRL performance and motor activity in the rat. Brain Res 642: 20-28.
Teegarden SL, Scott AN, Bale TL (2009). Early life exposure to a high fat diet promotes long-term changes in dietary preferences and central reward signaling. Neuroscience 162: 924-932.

Velazquez-Sanchez C, Ferragud A, Renau-Piqueras J, Canales JJ (2011). Therapeutic-like properties of a dopamine uptake inhibitor in animal models of amphetamine addiction. Int $J$ Neuropsychopharmacol 14: 655-665.

Volkow ND, Wang GJ, Tomasi D, Baler RD (2013). The addictive dimensionality of obesity. Biol Psychiatry 73: 811-818.

Volkow ND, Wise RA (2005). How can drug addiction help us understand obesity? Nat Neurosci 8: 555-560.

Wallace DL, Vialou V, Rios L, Carle-Florence TL, Chakravarty S, Kumar A et al (2008). The influence of DeltaFosB in the nucleus accumbens on natural reward-related behavior. J Neurosci 28: 10272-10277.

Werme M, Messer C, Olson L, Gilden L, Thoren P, Nestler EJ et al (2002). Delta FosB regulates wheel running. J Neurosci 22: 8133-8138.

Wiley JL, Compton AD, Golden KM (2000). Separation of drug effects on timing and behavioral inhibition by increased stimulus control. Exp Clin Psychopharmacol 8: 451-461.

Ziauddeen H, Farooqi IS, Fletcher PC (2012). Obesity and the brain: how convincing is the addiction model? Nat Rev Neurosci 13: $279-286$.

Supplementary Information accompanies the paper on the Neuropsychopharmacology website (http://www.nature.com/npp) 